\title{
The Role of Stem Cell Therapy in Alzheimer's Disease
}

\author{
Fatemeh Alipour ${ }^{1}$, Maryam Borhani Haghighi, ${ }^{1,2}$, Hoda Pasand Mojdeh ${ }^{3}$ \\ ${ }^{1}$ Shefa Neuroscience Research Center, Khatam Alanbia Hospital, Tehran, Iran \\ ${ }^{2}$ Department of Anatomy, School of Medicine, Tehran University of Medical Sciences, Tehran, Iran \\ ${ }^{3}$ School of Biology, College of Science, University of Tehran, Tehran, Iran
}

\section{A BSTRACT}

Introduction: Alzheimer's disease (AD) is the most ordinary reason of dementia in old population. $\mathrm{AD}$ is a permanent and progressive brain disorder that gradually deteriorates memory and speaking skills, and eventually leads to disability to accomplish the effortless skills. In this disease, the brain cells are gradually destroyed and as a result, the patients suffer from amnesia. Definitive treatment for $\mathrm{AD}$ has not been found yet and enormous efforts have been made to find novel and effective therapies. The stem cells are undifferentiated cells that have a potential to differentiate into many different cell types. Several studies revealed that neurons and glial cells have successfully been differentiated from various stem cells, such as neural, embryonic, and mesenchymal stem cells. Cell therapy is a potential novel therapeutic strategies for treatment of AD. Conclusion: Successful stem cell therapy in animal models of $\mathrm{AD}$ points to a potential therapeutic approach in patients with $\mathrm{AD}$.

*Corresponding Author: Maryam Borhani Haghighi

E-mail: borhanihm@gmail.com 
نقش درمانى سلولهاى بنيادى در بيمارى آلزايمر

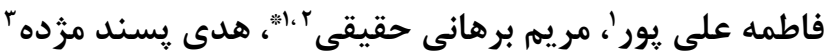 \\ 'مركز تحقيقات علوم اعصاب شفا، بيمارستان خاتم الانبياء، تهران، ايران

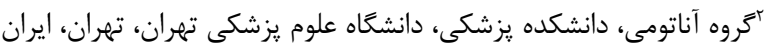

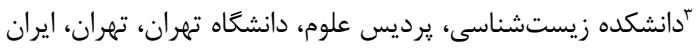

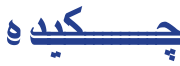

مقدمه: بيمارى آلزايمر دليل متداولترين زوال عقل در جمعيت سالخورده است. آلزايمر يك اختلال

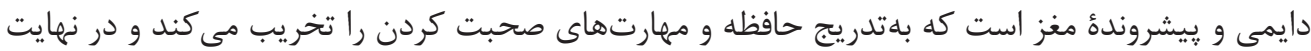

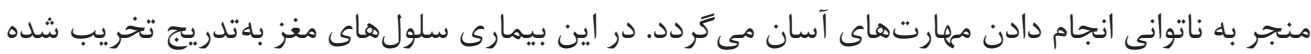

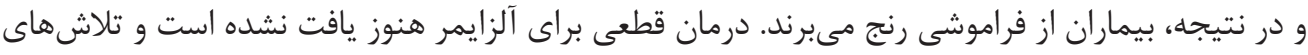

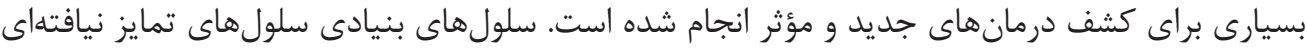

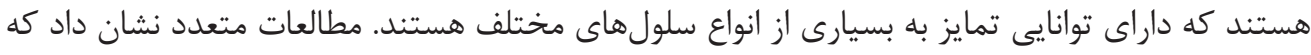

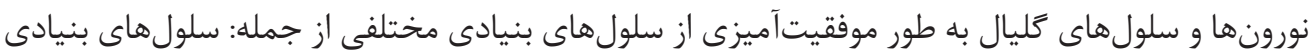

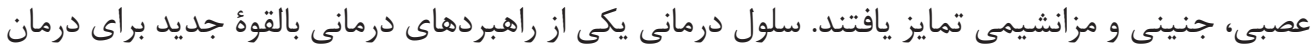

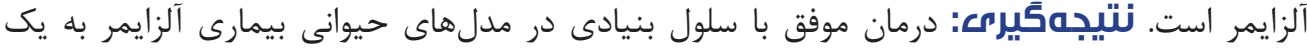

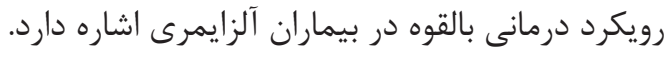

كليد وازهها:

ا. بيمارى آلزايمر

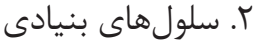
r. "r. fا. بافظه 


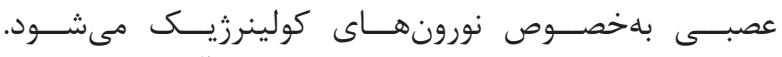

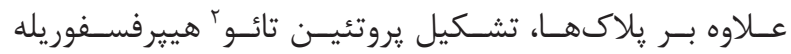

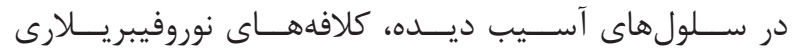

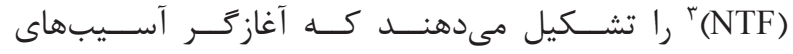

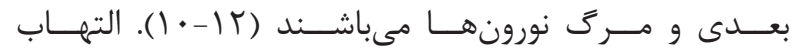

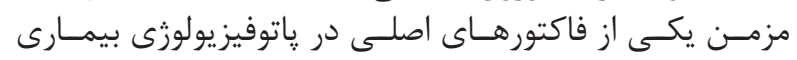

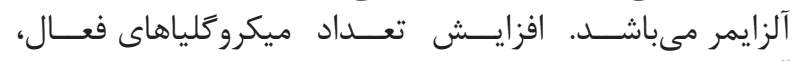

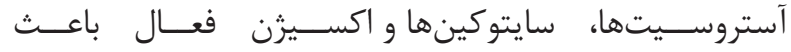

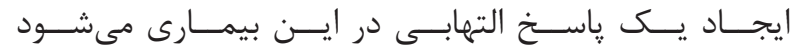

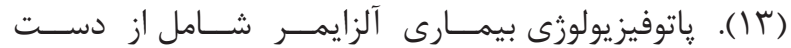

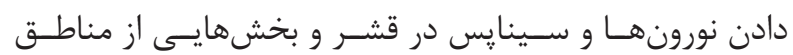

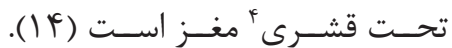
درمانهاى متداول بيمارى آلزايمر

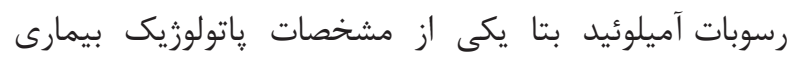

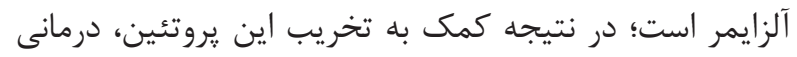

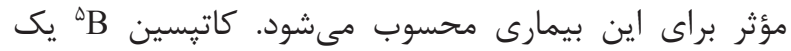

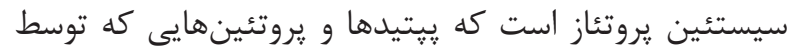

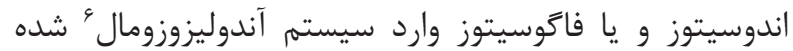

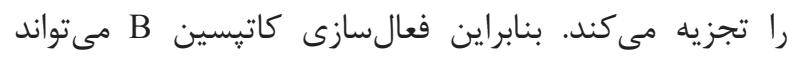

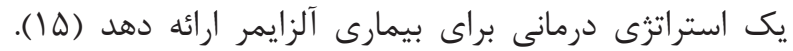

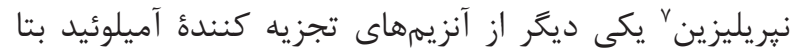

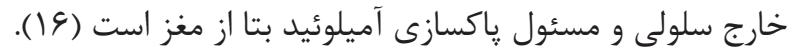

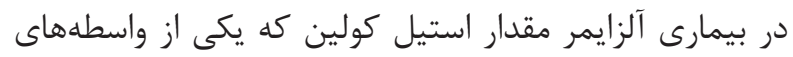
شيميايى مداخله

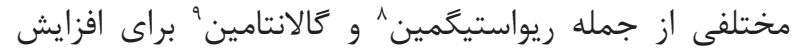

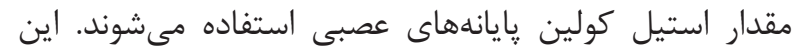

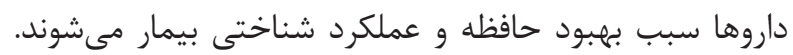

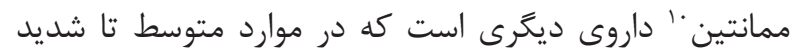

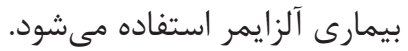

اين دارو نيز سبب بهتر شدن توانايى شناختى و حافظةٌ بيمار

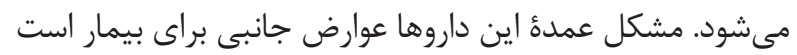

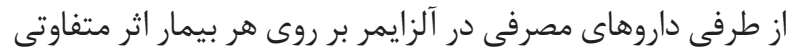

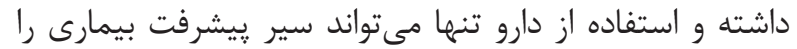
بكاهد و از شدت اختلال حافظه و مشكلات رفتارى إنى بيمار كم كند

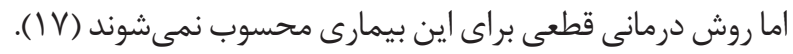

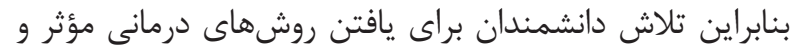

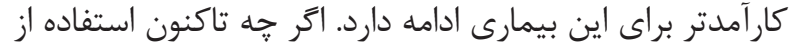

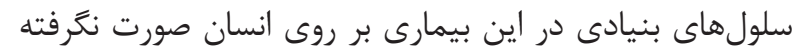

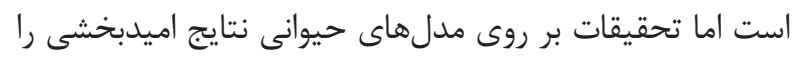

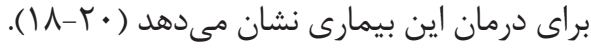

${ }^{1}$ Dementia

${ }^{2}$ Tau

${ }^{3}$ Neurofibrillary tangles

${ }^{4}$ Subcortical

${ }^{5}$ Cathepsin B
مقدمه

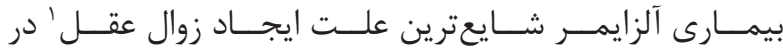

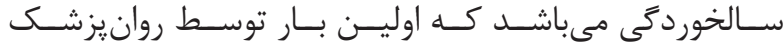

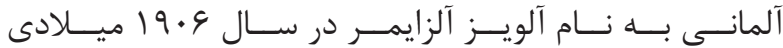

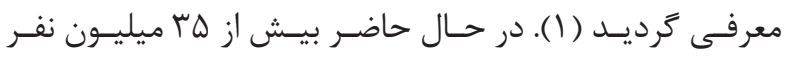

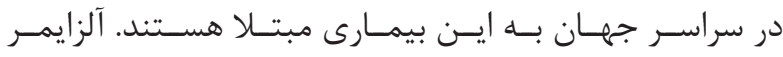

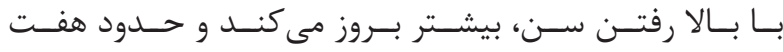

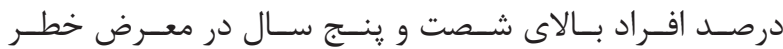

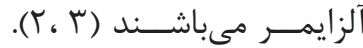

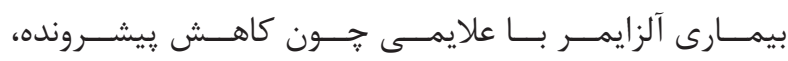

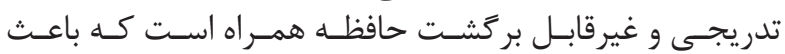

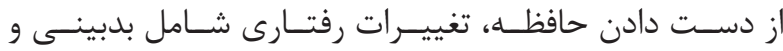

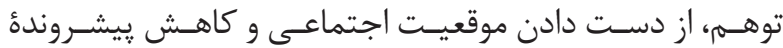

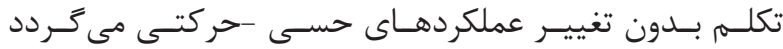

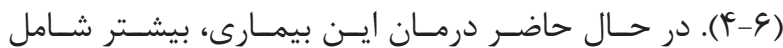

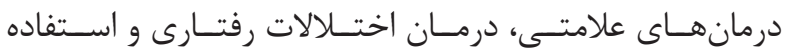

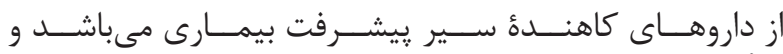

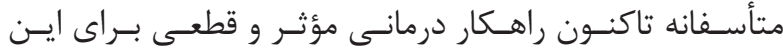

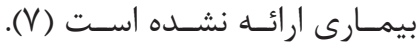

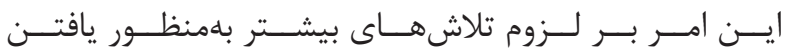

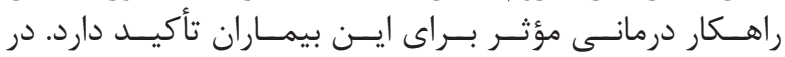

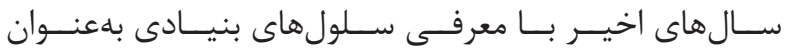

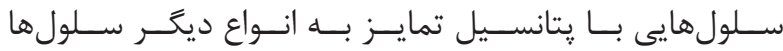

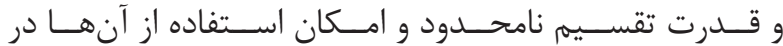

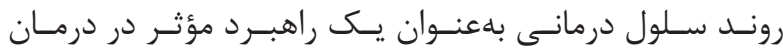

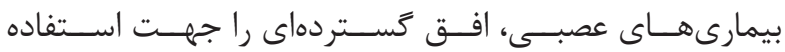

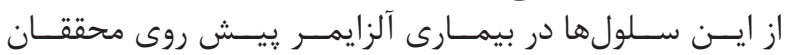

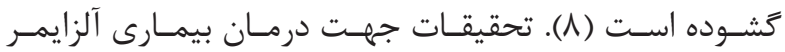

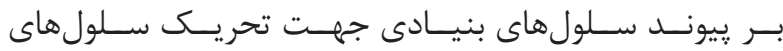

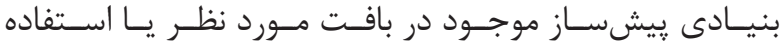

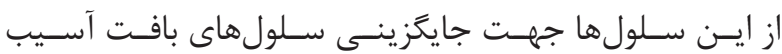

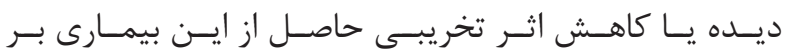

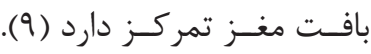
آسيبشناسى بيمارى آلزايمر

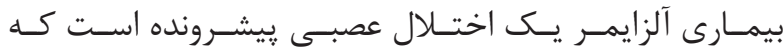

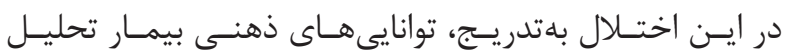

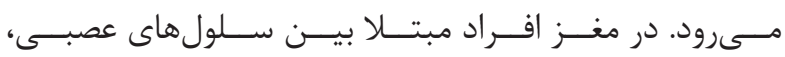

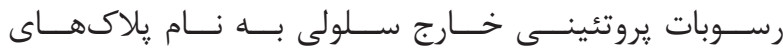

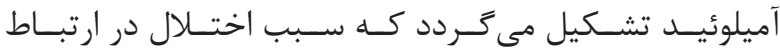

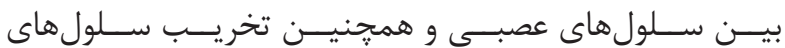

\footnotetext{
${ }^{6}$ Endolysosomal

${ }^{7}$ Neprilysin

${ }^{8}$ Rivastigmine

${ }^{9}$ Galantamine

${ }^{10}$ Memantine
} 
نقش داشته و قابلٍٍت پييوند به بافتهاى آسيبديده را دارند.

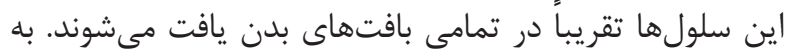

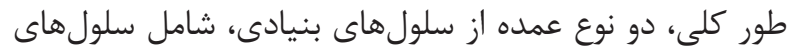

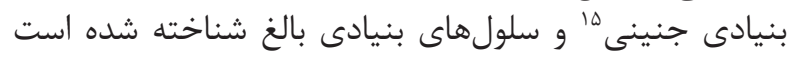

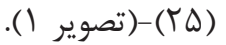

سلول هاى بنيادى خونساز "، بنيادى عصبى و بنيادى مزانشيمى

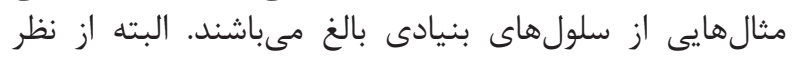

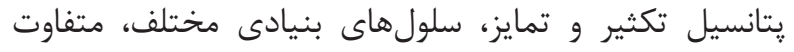

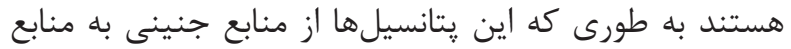

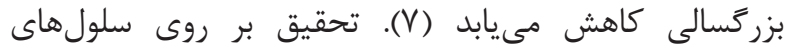

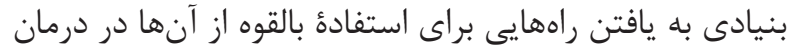

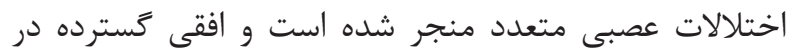

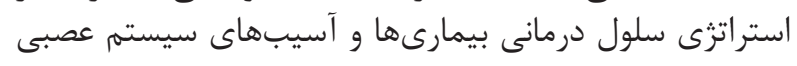

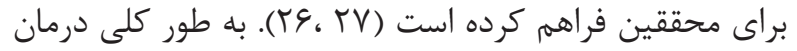

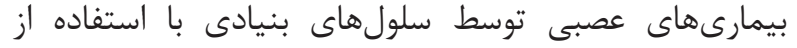
روشهاى جايخزينى سلولى، حفاظت عصبى عصى و انتقال زن رن انجام

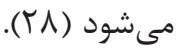

$$
\text { سلولهاى بنيادى جنينى }
$$

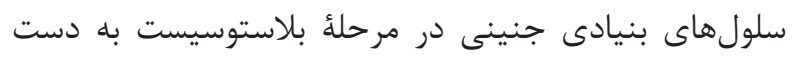

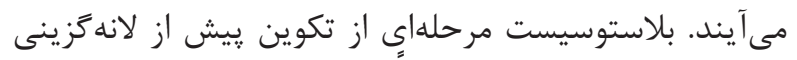

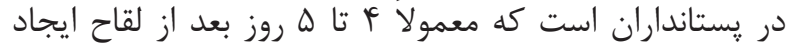

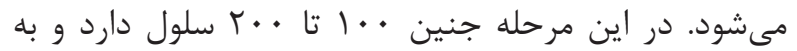

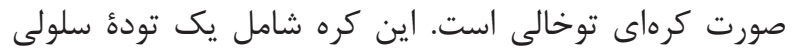

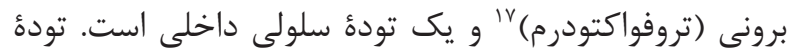

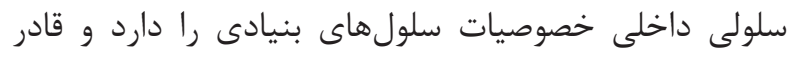

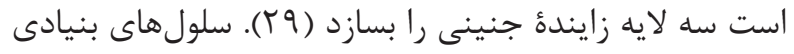

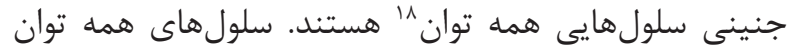

$$
\text { نورونزايى در بيمارى آلزايمر }
$$

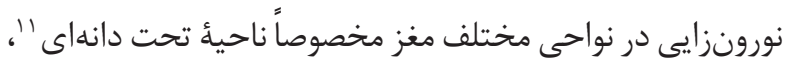

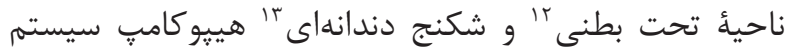

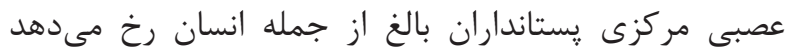

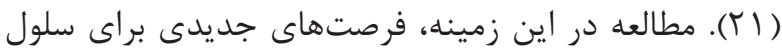

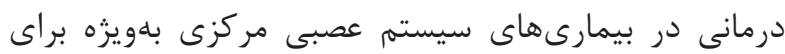

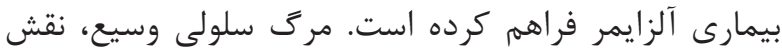

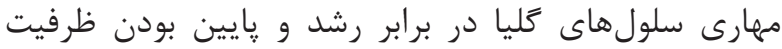

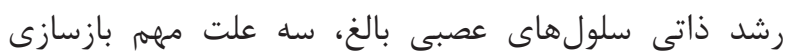

محدود در سيستم عصبى مركزى هـى هستند (Y) (Y).

اغلب تحقيقات صورت گرفته در مدلهاى آلزايمرى آميلوئيد

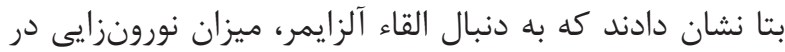

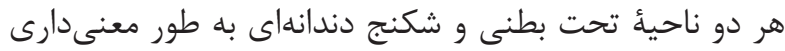

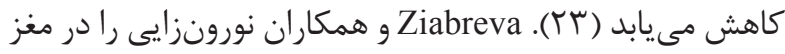

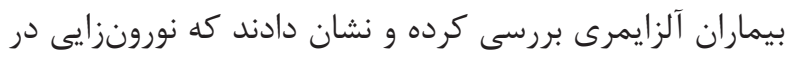

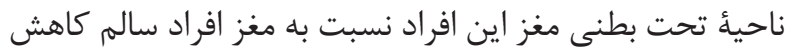

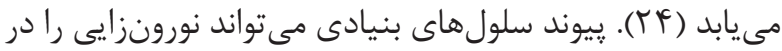

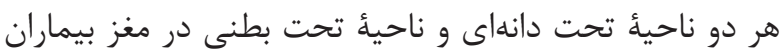

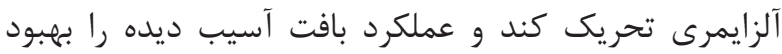

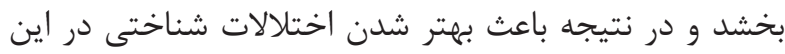

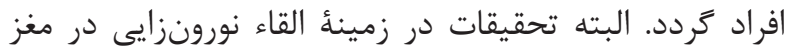

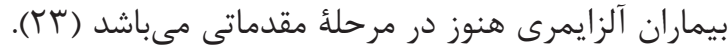

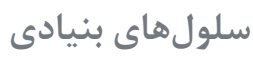

سلولهاى بنيادى، سلولهاى تخصص نيافته با قابليت تكثير،

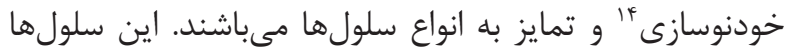

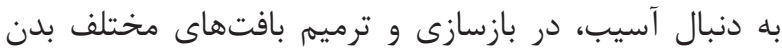

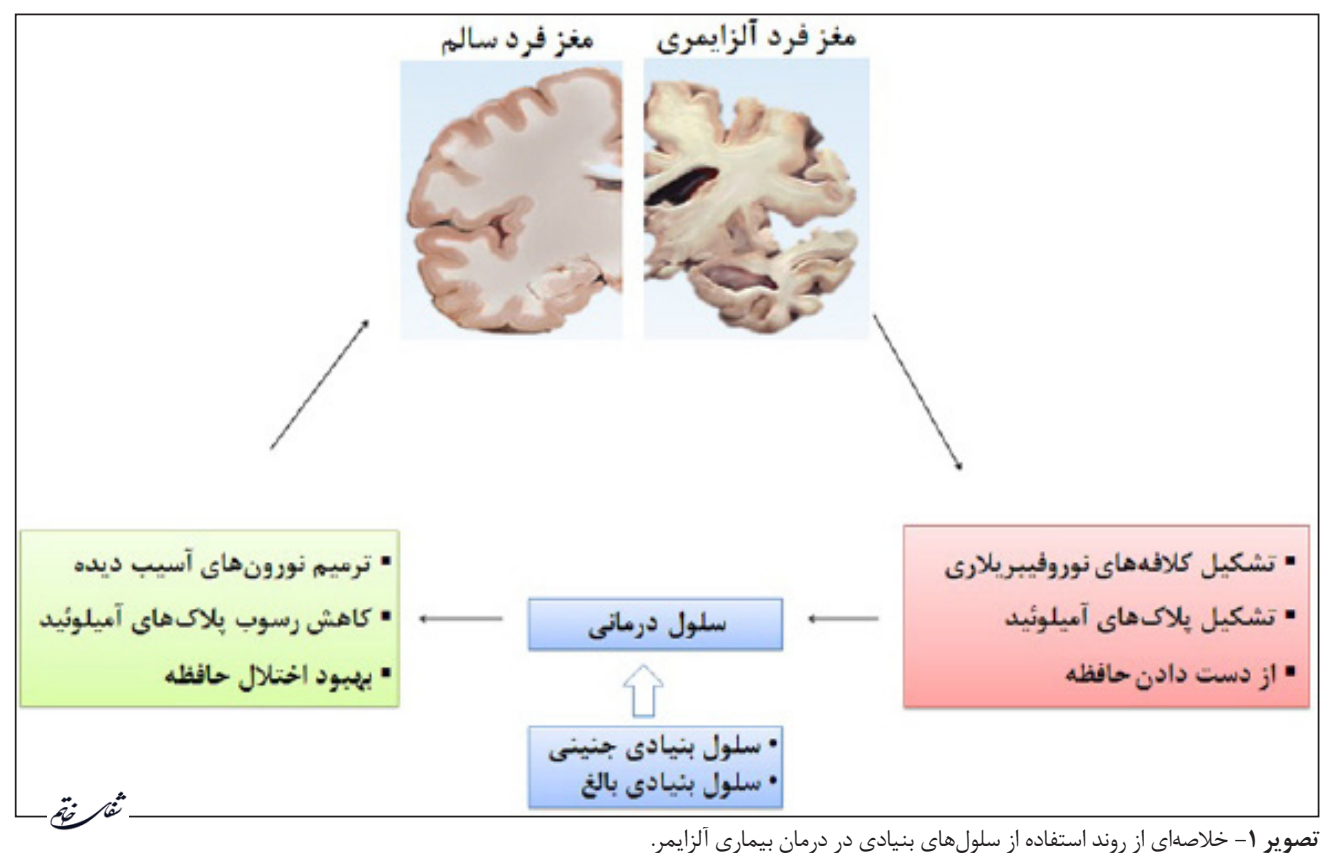

\footnotetext{
${ }^{11}$ Subgranular zone

${ }^{12}$ Subventricular zone

${ }^{13}$ Dentate gyrus

${ }^{14}$ Self-renewing
}

${ }^{15}$ Embryonic stem cell

${ }^{16}$ Hematopoietic stem cells

${ }^{17}$ Trophectoderm

${ }^{18}$ Totipotent 
حافظه قرار دارد. به همين دليل در صورتى كه بتوان از وارد آند

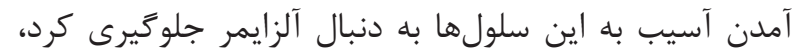

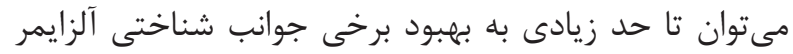

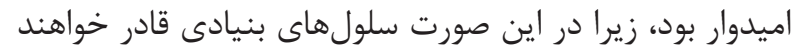

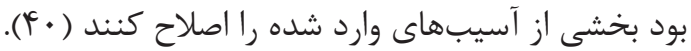

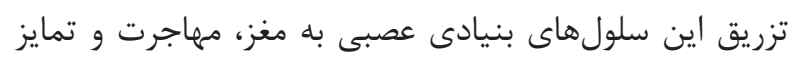

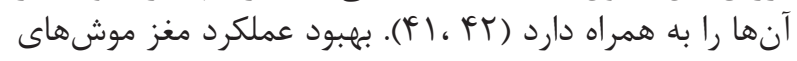

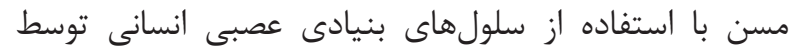

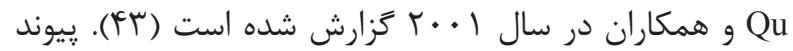

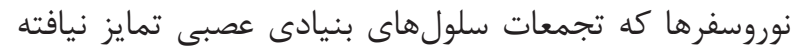

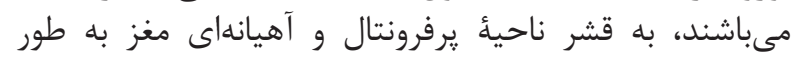

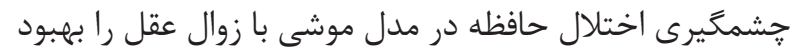

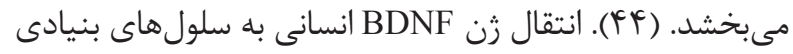

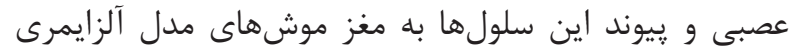

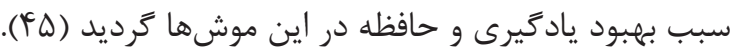

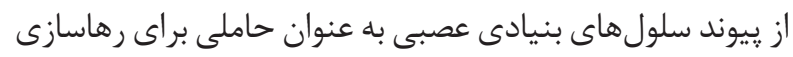

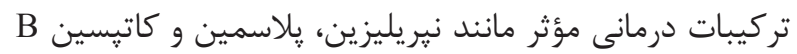

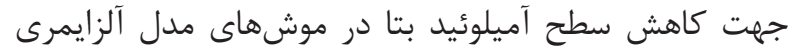

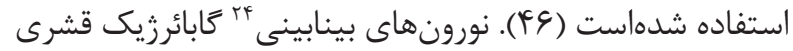
در برجستخى هاى كانغليونى ميانى (MGE)

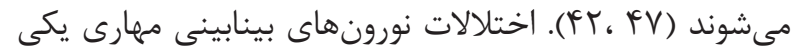

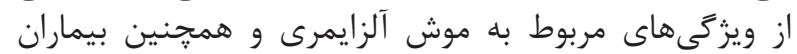

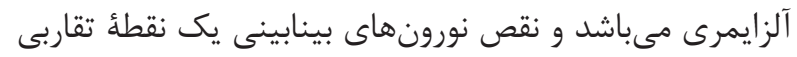

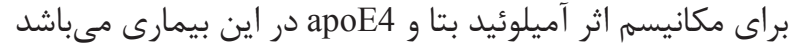

( $(\uparrow \wedge, 4 q)$

براى بررسى اينكه آيا با جايكزين شدن سلولهاى از دست

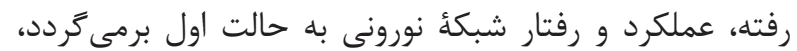

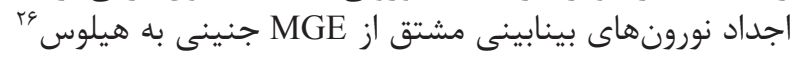

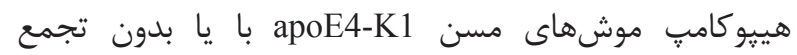

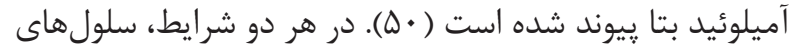

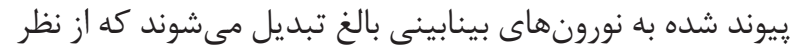

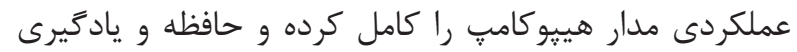

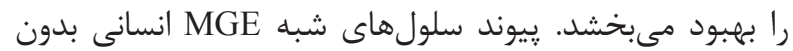

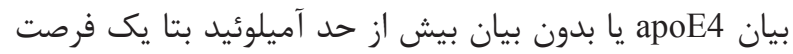

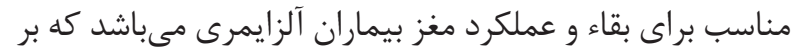

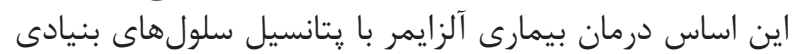

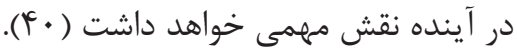
Ager

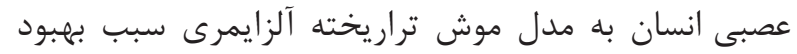

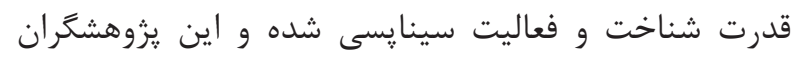

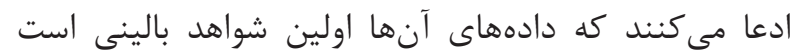

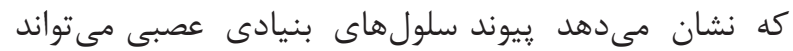

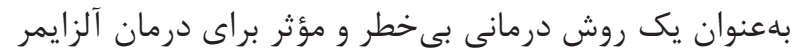

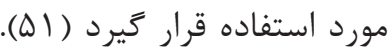

\section{${ }^{19}$ Escape latency}

${ }^{20}$ Teratoma

${ }^{21}$ Subventricular zone of lateral wall of lateral ventricle

${ }^{22}$ Hypothalamic subependymal zone
سلولهاى تمايزنيافتهاى هستند كه توانايى تبديل به انواع

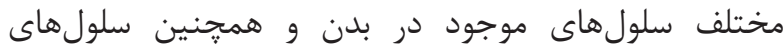
تشكيل دهنده جفت را دارا مى بـاشند.

در تعدادى از مطالعات يايه، مشخص شده است كه سلولهاى

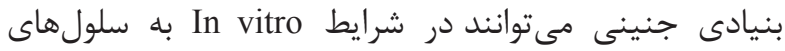

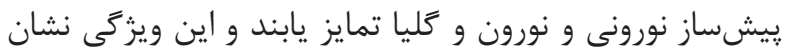

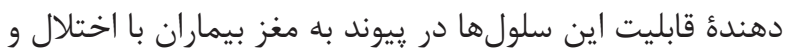

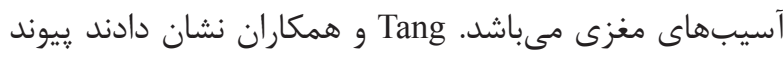

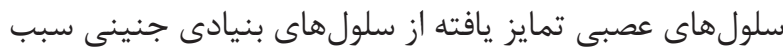

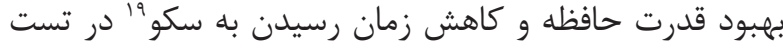

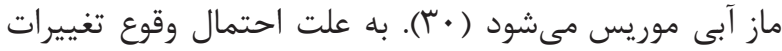

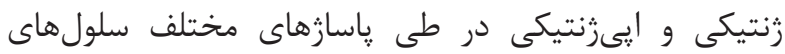

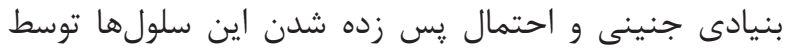

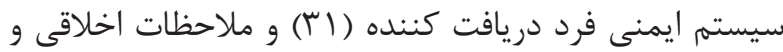

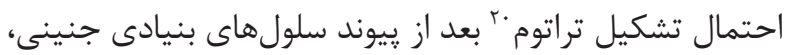

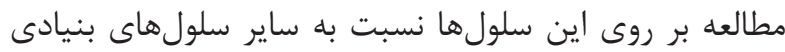

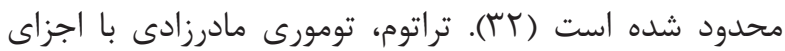

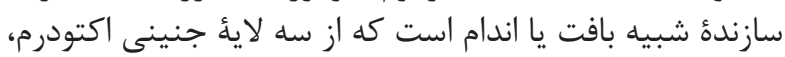
مزودرم و اندودرم منشاء مى ئيرد (سبّام).

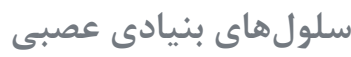
سالهاى بسيارى عقيده بر اين بود كه سيستم عصبى مركزِى

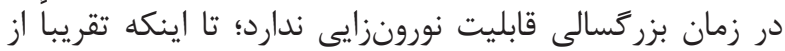

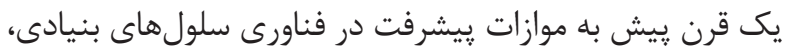

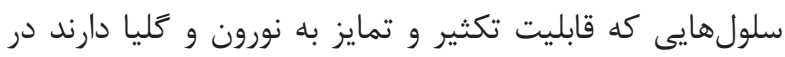

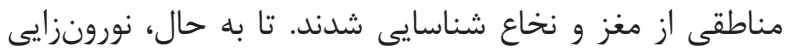

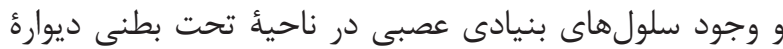

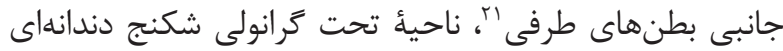

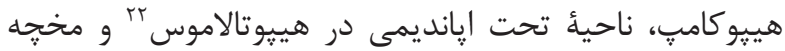

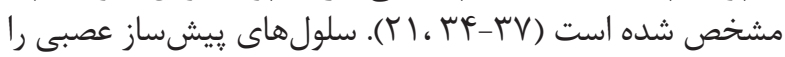

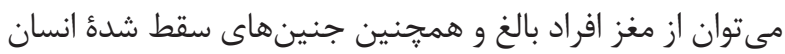

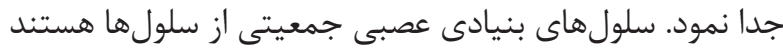

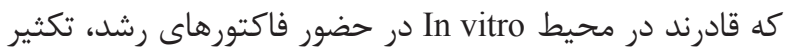

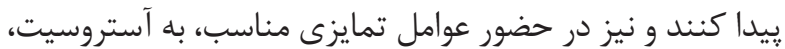

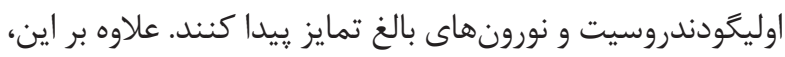

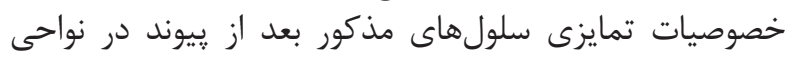

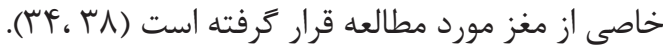

همجنين كزارش شده است سلولهايى كه حين جراحى هاى

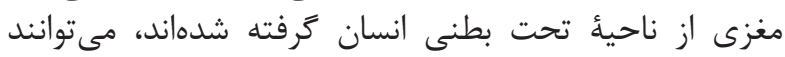

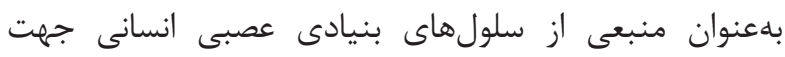

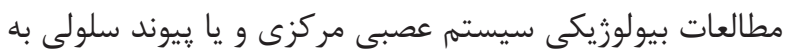

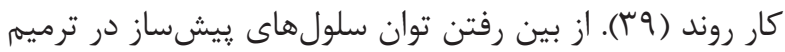

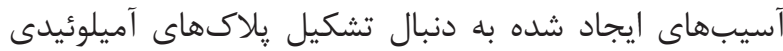

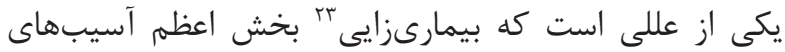

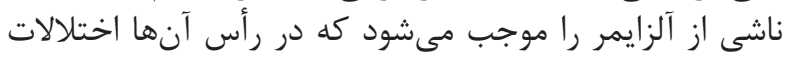

\footnotetext{
23. Pathogenesis

${ }^{24}$ Intermediate neurons

${ }^{25}$ Medial ganglionic eminence
}

${ }^{26}$ Hilus 
صورت داخل وريدى به موش مدل آلزايمرى تزريق شد، اين

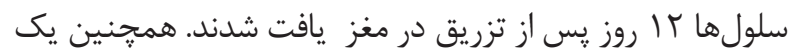

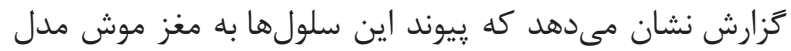

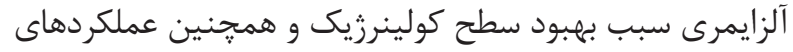

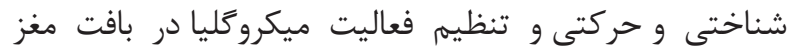

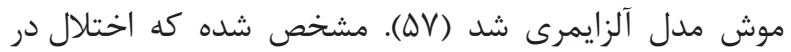

سيستم اتوفازى ممكن است به تجمع آميلوئيد بتا منجر شود.

سلولهاى بنيادى مزانشيمى به طور قابلتوجهى شكلى آيرى

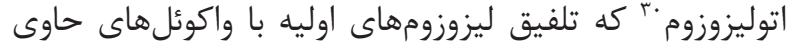

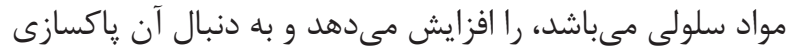

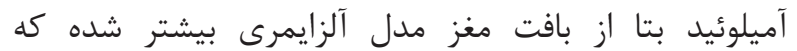

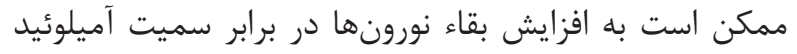

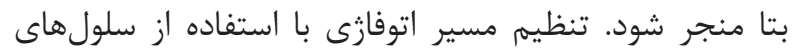

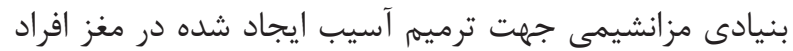

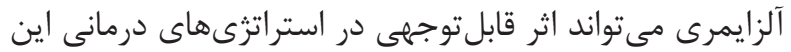

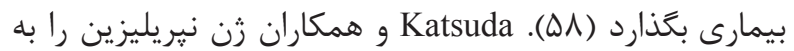

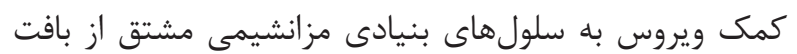

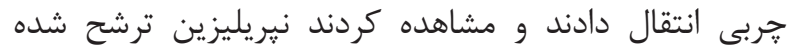

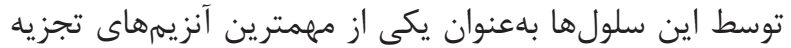

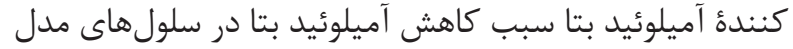

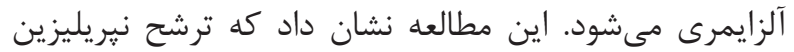

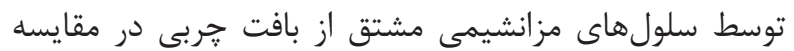

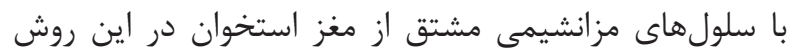
بيشتر است (ه9).

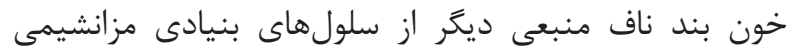

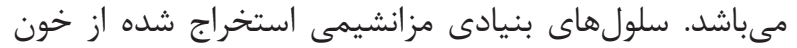

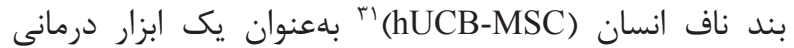

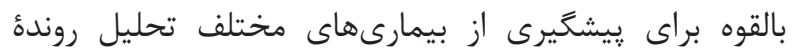

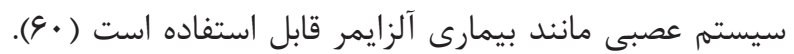

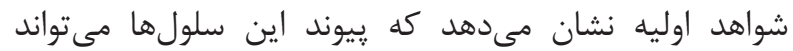

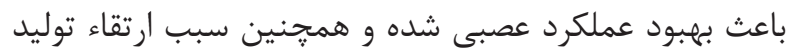

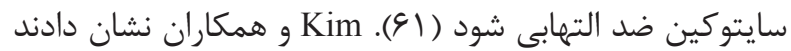

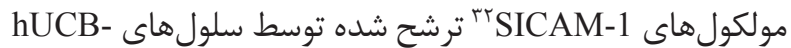

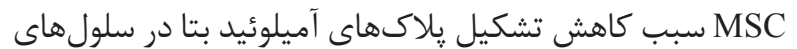

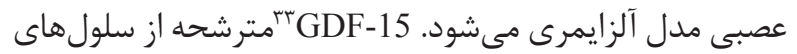

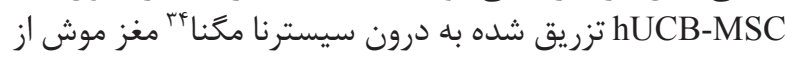

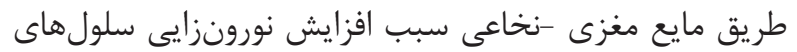

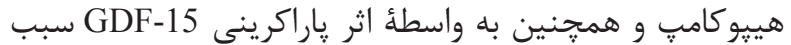

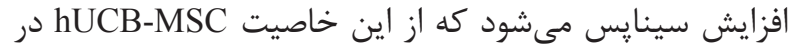

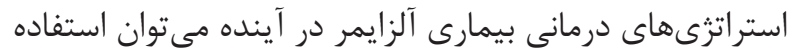

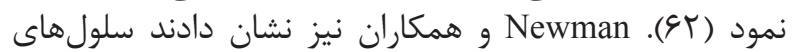

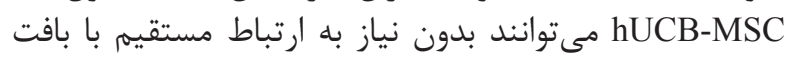
مغزى سبب ايجاد حمايت نورونى شوند (باند).

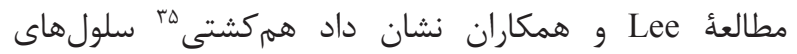

${ }^{27}$ Mesenchymal stem cells

${ }^{28}$ Interleukin 6

${ }^{29}$ Transforming growth factor beta 1

${ }^{30}$ Autolysosome

${ }^{31}$. Human umbilical cord blood- mesenchymal stem cells
با توجه به مطالعات متعدد انجام شده هنوز مشخص نيست

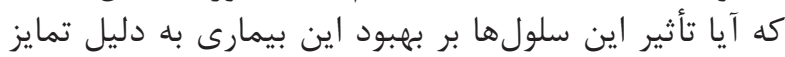

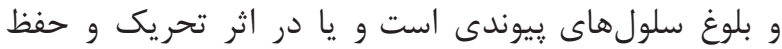

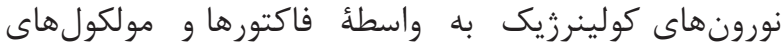

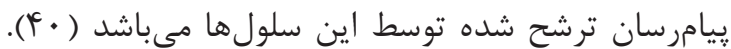

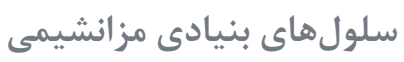

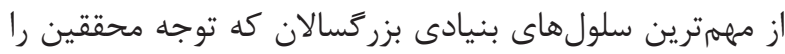

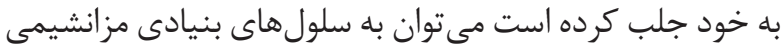

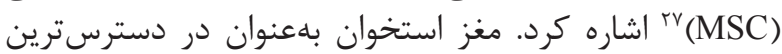

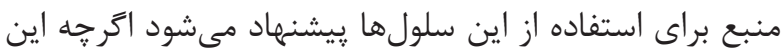

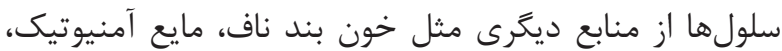

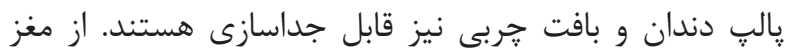

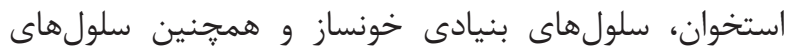

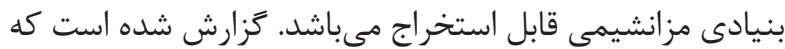

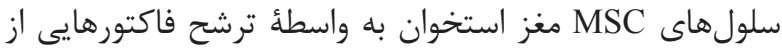

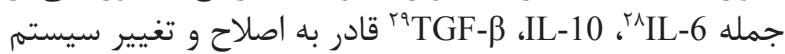

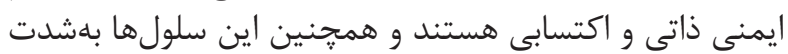

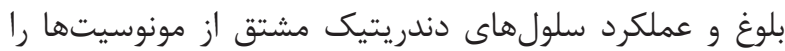

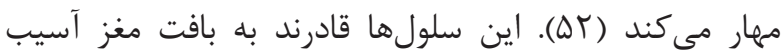

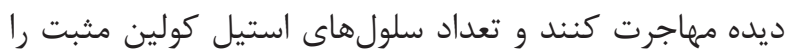

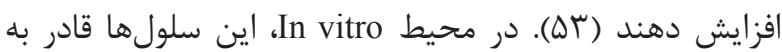

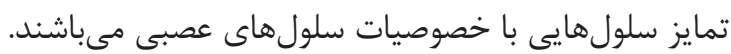

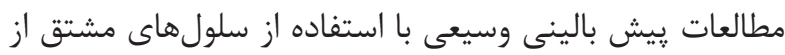

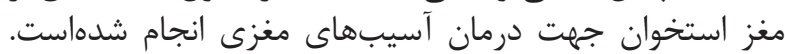

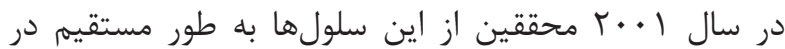

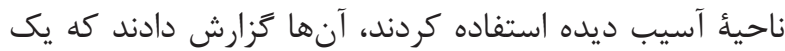

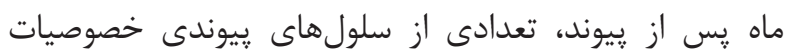

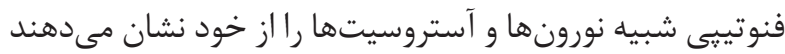

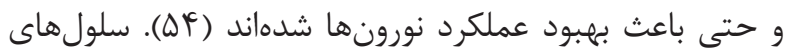

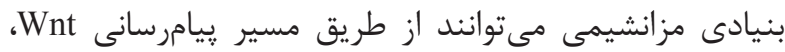

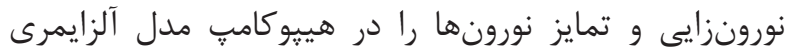

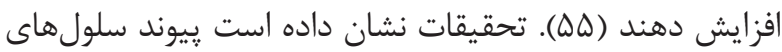

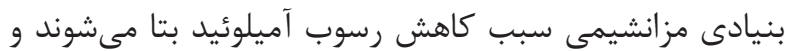

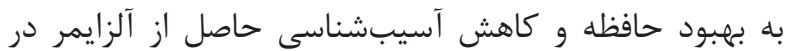

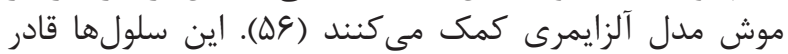

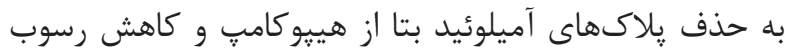

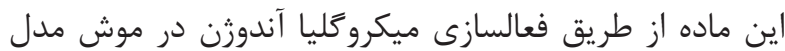
آلزايمرى نيز مىباشند (U) آهاري.

همانطور كه كَفته شد سلولهاى بنيادى مزانشيمى از منابع

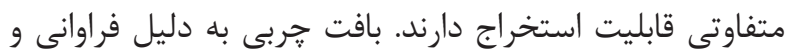

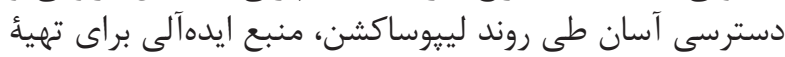
سلولهاى بنيادى مزانشيمى محسوب مى لئوسود. سلولهاى بنيادى مزانشيمى مشتق از بافت خربى انسان به مريه

32. Soluble intracellular adhesion molecule-1

33. Growth differentiation factor-15

34. Cisterna magna

35. Coculture 
سلولهاى بنيادى عصبى ييوند شده همجنين مى تواند تحت

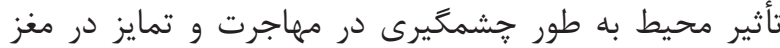

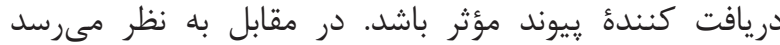

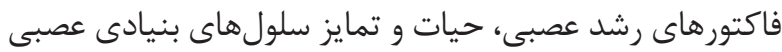

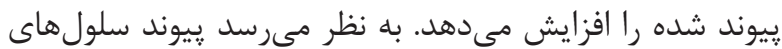

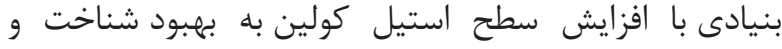

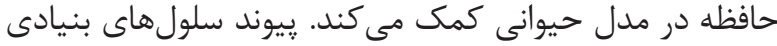

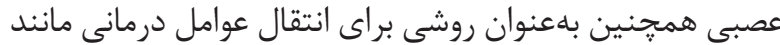

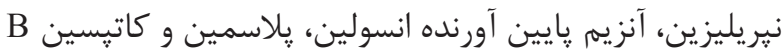

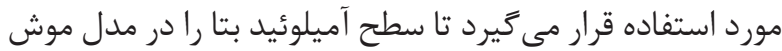
آلزايمرى كاهش دهد (14).

با ظهور تكنولوزى سلولهاى بنيادى و توانايى تبديل سلولهاى

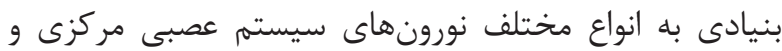

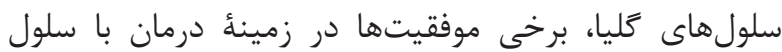

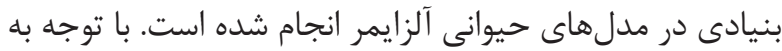

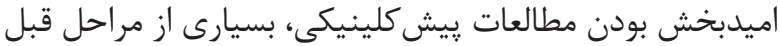

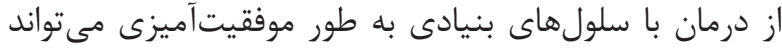

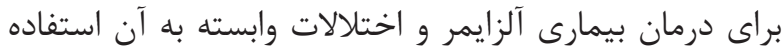

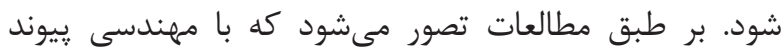

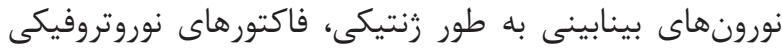

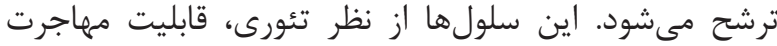

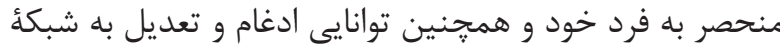

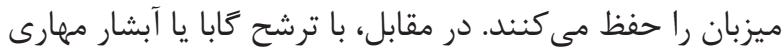

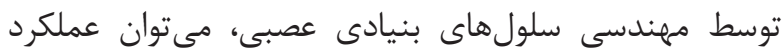
مهارى شبكههاى مغزى را تقويت كرد ( • (Y).

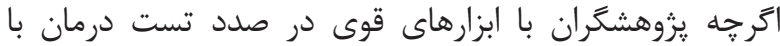

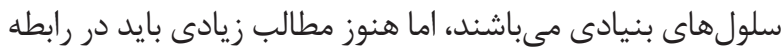

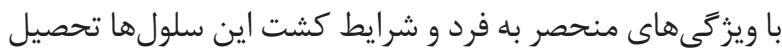

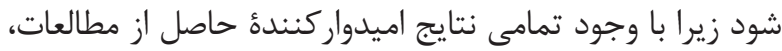

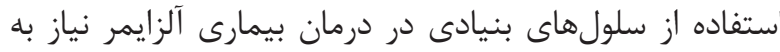

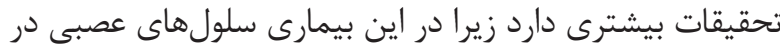

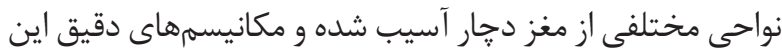
بيمارى هنوز به طور كامل مشخص مغر نشده است.

1. Tiraboschi P, Hansen L, Thal L, Corey-Bloom J. The importance of neuritic plaques and tangles to the development and evolution of AD. Neurology. 2004; 62(11): 1984-9.

2. Hirtz D, Thurman D, Gwinn-Hardy K, Mohamed M, Chaudhuri A, Zalutsky R. How common are the "common" neurologic disorders? Neurology. 2007; 68(5): 326-37.

3. Hebert LE, Scherr PA, Bienias JL, Bennett DA, Evans
مزانشيمى خون بند ناف با سلولهاى عصبى استخراج شده از

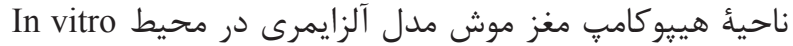

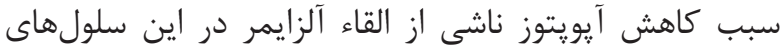

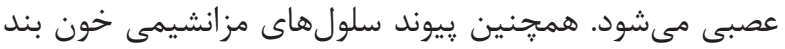

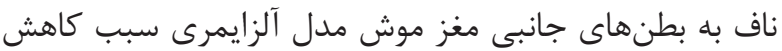

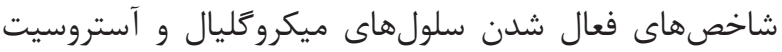

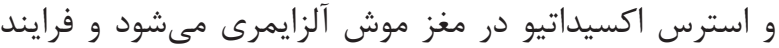

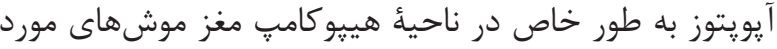

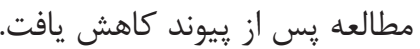

جالب توجه است كه در اين مطالعه نشان داده شد كه عملكرد

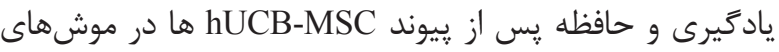

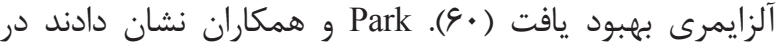

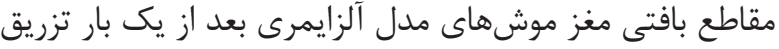

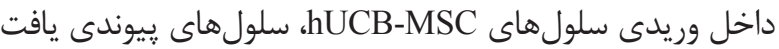

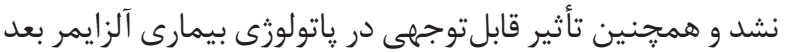

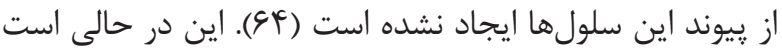

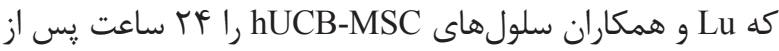
تروماى مغزى به صورت داخل وريدى به جوند إنان تزريق كردند

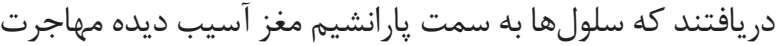

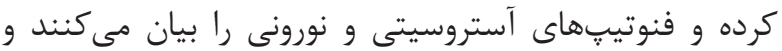

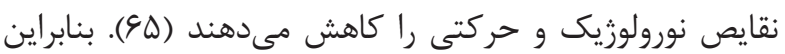

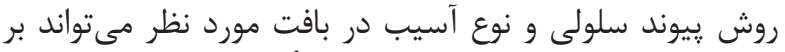

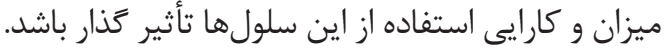

$$
\text { نتيجهَّيرى }
$$

استفاده از روش سلول درمانى جهت درمان بيمارى آلزايمر در

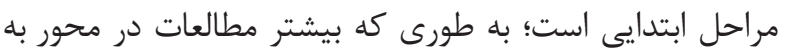

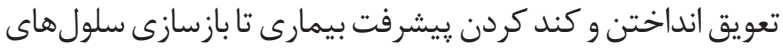

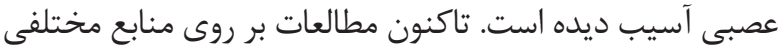

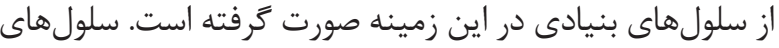

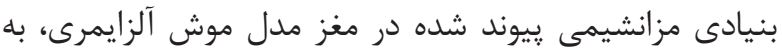

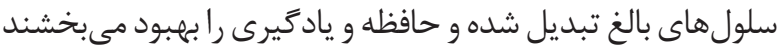
سو (9.9V)

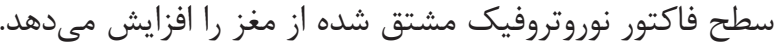

\section{منابع}

DA. Alzheimer disease in the US population: prevalence estimates using the 2000 census. Archives of neurology. 2003; 60(8): 1119-22.

4. Selkoe DJ. Alzheimer's disease: genes, proteins, and therapy. Physiol Rev. 2001; 81(2): 741-66.

5. Alipour F, Oryan S, Sharifzadeh M, Karimzadeh F, Kafami L, Irannejad $\mathrm{H}$, et al. The neuroprotective effect of a triazine derivative in an Alzheimer's rat model. Acta Med Iran. 2015; 53(1): 8-16. 
6. Alipour F, Karimzadeh F, Hasanzadeh G. P59: triazine improved hippocampal injuries in animal model of Alzheimer's disease. Shefaye Khatam. 2015; 2(S3): 109.

7. Koch P, Kokaia Z, Lindvall O, Brüstle O. Emerging concepts in neural stem cell research: autologous repair and cell-based disease modelling. Lancet Neurol. 2009; 8(9): 819-29.

8. Wray S, Fox NC. Stem cell therapy for Alzheimer's disease: hope or hype? Lancet Neurol. 2015. pii: S14744422(15)00382-8. doi: 10.1016/S1474-4422(15)00382-8.

9. Abdel-Salam OM. Stem cell therapy for Alzheimer's disease. CNS Neurol Disord Drug Targets. 2011; 10(4): 459-85.

10. Haass C, Selkoe DJ. Soluble protein oligomers in neurodegeneration: lessons from the Alzheimer's amyloid $\beta$-peptide. Nat Rev Mol Cell Biol. 2007; 8(2): 101-12.

11. Mohammadzadeh E, Alipour F, Khallaghi B. Evaluation of spatial memory impairment after intracerebroventricular streptozocin injection in adult rats. Shefaye Khatam. 2014; 2(1): 40-45.

12. Alipour F, Mohammadzadeh E, Khallaghi B. Evaluation of apoptosis in rat hippocampal tissue in an experimental model of Alzheimer's disease. Shefaye Khatam. 2014; 2(2): 13-20.

13. Münch G, Schinzel R, Loske C, Wong A, Durany $\mathrm{N}$, Li J, et al. Alzheimer's disease-synergistic effects of glucose deficit, oxidative stress and advanced glycation endproducts. J Neural Transm (Vienna). 1998; 105(4-5): 439-61.

14. Wenk GL. Neuropathologic changes in Alzheimer's disease: potential targets for treatment. J Clin Psychiatry. 2006; 67: 3-7.

15. Mueller-Steiner S, Zhou Y, Arai H, Roberson ED, Sun $\mathrm{B}$, Chen J, et al. Antiamyloidogenic and neuroprotective functions of cathepsin B: implications for Alzheimer's disease. Neuron. 2006; 51(6): 703-14.

16. Marr RA, Rockenstein E, Mukherjee A, Kindy MS, Hersh LB, Gage FH, et al. Neprilysin gene transfer reduces human amyloid pathology in transgenic mice. J Neurosci. 2003; 23(6): 1992-6.

17. Massoud F, Léger GC. Pharmacological treatment of Alzheimer disease. Can J Psychiatry. 2011; 56(10): 579-88.

18. Banerjee P, Sahoo A, Anand S, Bir A, Chakrabarti
S. The oral Iron chelator, deferasirox, reverses the agedependent alterations in Iron and amyloid- $\beta$ homeostasis in rat brain: implications in the therapy of Alzheimer's disease. J Alzheimers Dis. 2015; 49(3): 681-93.

19. Mury FB, da Silva WC, Barbosa NR, Mendes CT, Bonini JS, Sarkis JES, et al. Lithium activates brain phospholipase A2 and improves memory in rats: implications for Alzheimer's disease. Eur Arch Psychiatry Clin Neurosci. 2015; 11: 1-12.

20. Rodriguez-Perdigon M, Solas M, Moreno-Aliaga MJ, Ramirez MJ. Lipoic acid improves neuronal insulin signalling and rescues cognitive function regulating VGlut1 expression in high-fat-fed rats: Implications for Alzheimer's disease. Biochim Biophys Acta. 2016; 1862(4): 511-7.

21. Alvarez-Buylla A, Temple S. Stem cells in the developing and adult nervous system. J Neurobiol. 1998; 36(2): 105-10.

22. Purves D, Augustine G, Fitzpatrick D, Hall W, Lamantia A, Mcnamara J, et al. Epilepsy: the effect of pathological activity on neural circuitry. Purves D, Augustine G, Fitzpatrick D, Hall W, Lamantia A, Mcnamara J, et al. Neuroscience. 3th ed. Sinauer Associates, Inc. 2004; p. 600-1.

23. Lazarov O, Marr RA. Neurogenesis and Alzheimer's disease: at the crossroads. Exp Neurol. 2010; 223(2): 267-81.

24. Ziabreva I, Perry E, Perry R, Minger SL, Ekonomou A, Przyborski S, et al. Altered neurogenesis in Alzheimer's disease. J Psychosom Res. 2006; 61(3): 311-6.

25. Lindvall O, Kokaia Z, Martinez-Serrano A. Stem cell therapy for human neurodegenerative disorders-how to make it work. Nat Med. Nat Med. 2004; 10: 42-50.

26. Yee JZ, Oh K-W, Kim SH. Stem cell therapy for neurodegenerative diseases. Hanyang Medical Reviews. 2015; 35(4): 229-35.

27. Aligholi H, Hassanzadeh G, Rezayat M, Azari H, Ejtemaei Mehr S, Akbari M, et al. O36: threedimensional transplantation of adult neural stem cells in an acute brain injury model. Shefaye Khatam. 2015; 2(S3): 36-36.

28. Burgess A, Aubert I, Hynynen K. Delivery of stem cells to the brain: potential for treatment of neurodegenerative disease. J Ther Ultrasound. 2015; 3: O17. doi: 10.1186/2050-5736-3-S1-O17.

29. Bishop AE, Buttery LD, Polak JM. Embryonic stem 
cells. J Pathol. 2002; 197(4): 424-9.

30. Tang J, Xu H, Fan X, Li D, Rancourt D, Zhou G, et al. Embryonic stem cell-derived neural precursor cells improve memory dysfunction in $A \beta(1-40)$ injured rats. Neurosci Res. 2008; 62(2): 86-96.

31. Maroof AM, Keros S, Tyson JA, Ying S-W, Ganat YM, Merkle FT, et al. Directed differentiation and functional maturation of cortical interneurons from human embryonic stem cells. Cell stem cell. 2013; 12(5): 559-72.

32. Hentze H, Soong PL, Wang ST, Phillips BW, Putti TC, Dunn NR. Teratoma formation by human embryonic stem cells: evaluation of essential parameters for future safety studies. Stem Cell Res. 2009; 2(3): 198-210.

33. Thomson JA, Itskovitz-Eldor J, Shapiro SS, Waknitz MA, Swiergiel JJ, Marshall VS, et al. Embryonic stem cell lines derived from human blastocysts. science. 1998; 282(5391): 1145-7.

34. Temple S. The development of neural stem cells. Nature. 2001; 414(6859): 112-7.

35. Eriksson PS, Perfilieva E, Björk-Eriksson T, Alborn A-M, Nordborg C, Peterson DA, et al. Neurogenesis in the adult human hippocampus. Nat Med. 1998; 4(11): 1313-7.

36. Galli R, Gritti A, Bonfanti L, Vescovi AL. Neural stem cells an overview. Circ Res. 2003; 92(6): 598-608.

37. Reynolds BA, Weiss S. Generation of neurons and astrocytes from isolated cells of the adult mammalian central nervous system. Science. 1992; 255(5052): 1707-10.

38. Anderson DJ. Stem cells and pattern formation in the nervous system: the possible versus the actual. Neuron. 2001; 30(1): 19-35.

39. Ayuso-Sacido A, Roy NS, Schwartz TH, Greenfield JP, Boockvar JA. Long-term expansion of adult human brain subventricular zone precursors. Neurosurgery. 2008; 62(1): 223-9.

40. Tong LM, Fong H, Huang Y. Stem cell therapy for Alzheimer's disease and related disorders: current status and future perspectives. Exp Mol Med. 2015; 47(3): e151. doi: 10.1038/emm.2014.124.

41. Azari H, Sharififar S, Rahman M, Ansari S, Reynolds BA. Establishing embryonic mouse neural stem cell culture using the neurosphere assay. J Vis Exp. 2011; (47). pii: 2457. doi: 10.3791/2457.
42. Reubinoff BE, Itsykson P, Turetsky T, Pera MF, Reinhartz E, Itzik A, et al. Neural progenitors from human embryonic stem cells. Nat Biotechnol. 2001; 19(12): 1134-40.

43. Qu T, Brannen C, Kim H, Sugaya K. Human neural stem cells improve cognitive function of aged brain. Neuroreport. 2001; 12(6): 1127-32.

44. Wang Q, Matsumoto Y, Shindo T, Miyake K, Shindo A, Kawanishi M, et al. Neural stem cells transplantation in cortex in a mouse model of Alzheimer's disease. J Med Invest. 2006; 53(1-2): 61-9.

45. Zhao Z, Hu H, Feng G. Learning and memory amelioration of transplantation of the neural stem cells modified with human brain-derived neurotrophic factor gene on Alzheimer disease model rat. Zhongguo Xiu Fu Chong Jian Wai Ke Za Zhi. 2005; 19(5): 331-4.

46. Kim SU, Lee HJ, Kim YB. Neural stem cellbased treatment for neurodegenerative diseases. Neuropathology. 2013; 33(5): 491-504.

47. Tricoire L, Pelkey KA, Erkkila BE, Jeffries BW, Yuan X, McBain CJ. A blueprint for the spatiotemporal origins of mouse hippocampal interneuron diversity. J Neurosci. 2011; 31(30): 10948-70.

48. Huang Y, Mucke L. Alzheimer mechanisms and therapeutic strategies. Cell. 2012; 148(6): 1204-22.

49. Palop JJ, Mucke L. Amyloid-[beta]-induced neuronal dysfunction in Alzheimer's disease: from synapses toward neural networks. Nat Neurosci. 2010; 13(7): 812-8.

50. Tong LM, Djukic B, Arnold C, Gillespie AK, Yoon SY, Wang MM, et al. Inhibitory interneuron progenitor transplantation restores normal learning and memory in ApoE4 knock-in mice without or with $\mathrm{A} \beta$ accumulation. J Neurosci. 2014; 34(29): 9506-15.

51. Ager RR, Davis JL, Agazaryan A, Benavente F, Poon WW, LaFerla FM, et al. Human neural stem cells improve cognition and promote synaptic growth in two complementary transgenic models of Alzheimer's disease and neuronal loss. Hippocampus. 2015; 25(7): 813-26.

52. Aggarwal S, Pittenger MF. Human mesenchymal stem cells modulate allogeneic immune cell responses. Blood. 2005; 105(4): 1815-22.

53. Salem AM, Ahmed HH, Atta HM, Ghazy MA, Aglan HA. Potential of bone marrow mesenchymal stem cells in management of Alzheimer's disease in female rats. Cell Biol Int. 2014; 38(12): 1367-83. 
54. Camberlain G, Fox J, Ashton B, Middleton J. Mesenchymal stem cells: their phenotype, differentiation capacity, immunological features, and potential for homing. Stem Cells. 2007; 25(11): 2739-49.

55. Oh SH, Kim HN, Park H-J, Shin JY, Lee PH. Mesenchymal stem cells increase hippocampal neurogenesis and neuronal differentiation by enhancing the wnt signaling pathway in an Alzheimer's disease model. Cell Transplant. 2015; 24(6): 1097-109.

56. Choi SS, Lee S-R, Kim SU, Lee HJ. Alzheimer's disease and stem cell therapy. Exp Neurobiol. 2014; 23(1): 45-52.

57. Park D, Yang G, Bae DK, Lee SH, Yang YH, Kyung $\mathrm{J}$, et al. Human adipose tissue-derived mesenchymal stem cells improve cognitive function and physical activity in ageing mice. J Neurosci Res. 2013; 91(5): 660-70.

58. Shin JY, Park HJ, Kim HN, Oh SH, Bae J-S, Ha $\mathrm{H}-\mathrm{J}$, et al. Mesenchymal stem cells enhance autophagy and increase $\beta$-amyloid clearance in Alzheimer disease models. Autophagy. 2014; 10(1): 32-44.

59. Katsuda T, Tsuchiya R, Kosaka N, Yoshioka Y, Takagaki K, Oki K, et al. Human adipose tissue-derived mesenchymal stem cells secrete functional neprilysinbound exosomes. Sci Rep. 2013; 3: 1197. doi: 10.1038/ srep01197.

60. Lee HJ, Lee JK, Lee H, Shin J-w, Carter JE, Sakamoto T, et al. The therapeutic potential of human umbilical cord blood-derived mesenchymal stem cells in Alzheimer's disease. Neurosci Lett. 2010; 481(1): 30-5.

61. Liu W-S, Chen C-T, Foo N-H, Huang H-R, Wang J-J, Chen S-H, et al. Human umbilical cord blood cells protect against hypothalamic apoptosis and systemic inflammation response during heatstroke in rats. Pediatr Neonatol. 2009; 50(5): 208-16.

62. Kim DH, Lee D, Chang EH, Kim JH, Hwang JW, Kim J-Y, et al. GDF-15 secreted from human umbilical cord blood mesenchymal stem cells delivered through the cerebrospinal fluid promotes hippocampal neurogenesis and synaptic activity in an Alzheimer's disease model. Stem Cells Dev. 2015; 24(20): 2378-90.

63. Newman MB, Willing AE, Manresa JJ, DavisSanberg C, Sanberg PR. Stroke-induced migration of human umbilical cord blood cells: time course and cytokines. Stem Cells Dev. 2005; 14(5): 576-86.

64. Park SE, Lee NK, Lee J, Hwang JW, Choi SJ, Hwang $\mathrm{H}$, et al. Distribution of human umbilical cord bloodderived mesenchymal stem cells in the Alzheimer's disease transgenic mouse after a single intravenous injection. Neuroreport. 2016; 27(4): 235-41. doi: 10.1097/WNR.0000000000000526.

65. Lu D, Sanberg PR, Mahmood A, Li Y, Wang L, Sanchez-Ramos J, et al. Intravenous administration of human umbilical cord blood reduces neurological deficit in the rat after traumatic brain injury. Cell Transplant. 2002; 11(3): 275-81.

66. Lee HJ, Kim KS, Kim EJ, Choi HB, Lee KH, Park $\mathrm{IH}$, et al. Brain transplantation of immortalized human neural stem cells promotes functional recovery in mouse intracerebral hemorrhage stroke model. Stem cells. 2007; 25(5): 1204-12.

67. Yamasaki TR, Blurton-Jones M, Morrissette DA, Kitazawa M, Oddo S, LaFerla FM. Neural stem cells improve memory in an inducible mouse model of neuronal loss. J Neurosci 2007; 27(44): 11925-33. 